\title{
Promoción de mecanismos de prevención y solución de conflictos para la eficacia en la protección de los consumidores
}

\section{Carolina Aquise Niño de Guzmán}

Instituto Nacional de Defensa de la Competencia y de la Protección de la Propiedad Intelectual, Lima, Perú

Recibido: 28/8/2018 / Aprobado: 31/1/2019

doi: 10.26439/iusetpraxis2018.n48-49.4499

Resumen. El presente artículo busca analizar los objetivos de la actual política nacional de protección al consumidor y su importancia como instrumentos para hacer viable la función del Estado en la protección de los derechos de los consumidores, en el régimen constitucional vigente. Sobre esta base, se examina la función de Indecopi en la ejecución de dichos objetivos, mediante la promoción de mecanismos de prevención y solución de conflictos entre proveedores y consumidores.

PALABRAS CLAVE: simplificación / solución de conflictos / conciliación / arbitraje / Libro de Reclamaciones / programa de cumplimiento normativo

\section{Promotion of mechanisms for conflict prevention} and resolution to attain an effective consumer protection

Авstract. The present article pursues to analyze the objectives of the current national consumer protection policy and its importance as an instrument that facilitate the government's duty to protect the consumers' rights according to the constitutional regime in force. In such context, this paper discusses the role of the National Institute for the Defense of Competition and Intellectual Property (Indecopi) regarding the accomplishment of said objectives through the promotion of mechanisms for conflict prevention and resolution between suppliers and consumers.

KEYWORDS: simplification / conflict resolution / conciliation / arbitration / Complaint Book / regulatory compliance program 


\section{INTRODUCCIÓN ${ }^{1}$}

El 30 de diciembre del 2016 se publicó el Decreto Legislativo 1308, que modificó diversas disposiciones del Código de Protección y Defensa del Consumidor (en adelante, el Código), Ley 29571, con la finalidad de simplificar y otorgar celeridad a los procedimientos administrativos de protección al consumidor, de manera que permitan un pronunciamiento oportuno de la autoridad administrativa y una solución eficaz a las controversias de consumo.

En consonancia con el objetivo de promover diversas medidas destinadas a brindar una solución más eficaz a los conflictos entre consumidores y proveedores, el Consejo Nacional de Protección del Consumidor $^{2}$ impulsó la elaboración de la Política Nacional de Protección al Consumidor (en adelante, la Política Nacional), la cual fue aprobada por la Presidencia del Consejo de Ministros (PCM) mediante Decreto Supremo 006-2017-PCM. Asimismo, el Decreto Supremo 24-2017-PCM estableció el Plan Nacional de Protección de los Consumidores 2017-2020, como instrumento para la implementación de la Política Nacional y con el fin de lograr una mayor y más eficaz protección de los consumidores o usuarios en el país, con una visión a largo plazo.

Considerando la implementación de las modificaciones e instrumentos antes descritos, el presente trabajo busca identificar cuáles son los objetivos estratégicos dentro de la Política Nacional y la importancia de la función de Indecopi en la ejecución de esta visión. Para ello, se examinan las últimas modificaciones de simplificación administrativa aplicables a los procedimientos de protección al consumidor que están bajo su competencia.

1 La autora señala que toda opinión vertida en el presente artículo le es atribuible únicamente a título personal y que de ningún modo involucra parecer alguno de la institución en la que participa como profesional. Asimismo, agradece los comentarios del señor Alberto Cunya Merino por haber enriquecido el presente artículo.

2 El Consejo Nacional de Protección del Consumidor fue creado por el artículo 133 del Código como órgano de coordinación presidido por el Instituto Nacional de Defensa de la Competencia y de la Protección de la Propiedad Intelectual (Indecopi). Está conformado por representantes de diversos sectores del Poder Ejecutivo, de los gobiernos regionales y locales, de los organismos reguladores y de la sociedad civil, entre otros. 


\section{El DEBER DEl Estado Frente A los CONSUMidores}

Sin duda alguna, al revisar el esquema constitucional peruano, podemos observar que ha optado por una economía social de mercado que implica, por un lado, la promoción de la iniciativa privada en un marco de libre competencia y, por otro lado, un Estado garante del bienestar social - es decir, empleos productivos, trabajo digno, reparto justo del ingreso-, que, en ese sentido, está interesado en defender y proteger a los consumidores (Donayre Montesinos, 2015, pp. 54-55). Así, cabe recordar que el artículo 65 de la Constitución establece que el Estado defiende el interés de los consumidores y usuarios. Con respecto a este supuesto normativo, el Tribunal Constitucional ha señalado lo siguiente en la sentencia del Expediente 01865-2010-PA/TC:

12. El artículo 65 de la Constitución prescribe la defensa de los consumidores y usuarios a través de un derrotero jurídico binario, a saber: (a) establece un principio rector para la actuación del Estado; y (b) consagra un derecho personal y subjetivo.

13. En el primer ámbito, el artículo 65 de la Constitución expone una pauta basilar o postulado destinado a orientar y fundamentar la activación del Estado respecto a cualquier actividad económica. Así, el juicio estimativo y el juicio lógico derivado de la conducta del Estado sobre la materia tienen como horizonte tuitivo la defensa de los intereses de los consumidores y usuarios.

14. En el segundo ámbito, el artículo 65 de la Constitución reconoce la facultad de acción defensiva de los consumidores y usuarios en los casos de transgresión o desconocimiento de sus legítimos intereses; es decir, reconoce y apoya el atributo de exigir al Estado una actuación determinada cuando se produzca alguna forma de amenaza o afectación efectiva de los derechos del consumidor o del usuario, incluyendo la capacidad de acción contra el propio proveedor. (Sentencia 018652010, 2011)

Lo anterior supone que el Estado debe destinar su actuación considerando la defensa de los intereses de los consumidores y usuarios. En esta línea, el vigente Código y sus normas modificatorias establecen las reglas de protección mínima para la defensa de los consumidores y, asimismo, definen las políticas públicas que servirán como expresión del propósito y acción del Estado en materia de protección al consumidor. 


\section{ObJetivos de la POlítica NACIONAL de PROTECCIÓN AL CONSUMIDOR}

Mediante el Decreto Supremo 006-2017-PCM, la PCM aprobó la Política Nacional que tomaba en cuenta la evaluación de diversos factores del entorno social actual en donde se generan las relaciones de consumo. Como resultado de este análisis, este instrumento instauró un objetivo general y cuatro (4) objetivos específicos que orientan la actuación del Estado en materia de protección al consumidor.

El objetivo general de la Política Nacional establece lo siguiente:

Objetivo general: el objetivo de la Política Nacional de Protección y Defensa del Consumidor es contribuir a un mayor y más eficaz nivel de protección de los derechos de los consumidores con equidad y con mayor incidencia en los sectores de consumidores más vulnerables, debiendo para ello consolidar e integrar de modo progresivo el Sistema Nacional Integrado de Protección del Consumidor. (Decreto Supremo 006-2017-PCM, 2017)

Como se puede ver, en el objetivo general, el Estado considera prioritario lograr una mayor y más eficaz protección de los derechos de los consumidores. Esta visión parte, acertadamente, del reconocimiento de un entorno social más dinámico y con consumidores más empoderados, quienes utilizan las redes sociales y otras tecnologías para difundir al instante cualquier problema en sus relaciones de consumo y exigen no solo una solución a sus conflictos, sino también que esta se produzca de forma más eficaz.

Además, la Política Nacional reconoce la existencia de dos factores relevantes del entorno social actual: (a) la tendencia creciente de la población adulta mayor y (b) una alta tasa de población aún analfabeta o con poca instrucción. Ambos corresponden a un mayor segmento de consumidores en situación de vulnerabilidad frente a las relaciones de consumo, por lo que la Política Nacional también se instaura con el objetivo de incidir en la protección de los consumidores más vulnerables.

Por otra parte, los cuatro (4) objetivos específicos que guían la actuación de las entidades con competencia en materia de protección al consumidor son estos:

Objetivos específicos:

a) Fortalecer la educación de los agentes del mercado (consumidores y proveedores) con relación a los derechos de los consumidores y 
su obligatorio cumplimiento, implementando las actividades dirigidas a la orientación de los consumidores en el ejercicio de sus derechos y la difusión de los mismos.

b) Garantizar la seguridad de los consumidores en el marco de las relaciones de consumo.

c) Implementar mecanismos de prevención y solución de conflictos de consumo entre proveedores y consumidores.

d) Fortalecer el Sistema Nacional Integrado de Protección al Consumidor. (Decreto Supremo 006-2017-PCM, 2017)

A partir de estos objetivos específicos, la Política Nacional se ha estructurado y organizado sobre la base de cuatro (4) ejes estratégicos: (i) educación, orientación y difusión; (ii) protección de la salud y seguridad de los consumidores; (iii) mecanismos de prevención y solución de conflictos entre proveedores y consumidores; y (iv) fortalecimiento del Sistema Nacional Integrado de Protección al Consumidor. Entre los ejes estratégicos de la Política Nacional, cabe destacar que uno de ellos menciona el papel del Estado como promotor de mecanismos eficaces de prevención y solución de conflictos entre proveedores y consumidores, lo cual se encuentra en sintonía con el objetivo general de brindar una protección más eficaz a los consumidores y, a su vez, coincide con lo previsto en el artículo IV del Código:

Artículo IV.- Políticas públicas

El Estado garantiza mecanismos eficaces y expeditivos para la solución de conflictos entre proveedores y consumidores. Para tal efecto, promueve que los proveedores atiendan y solucionen directa y rápidamente los reclamos de los consumidores, el uso de mecanismos alternativos de solución como la mediación, la conciliación y el arbitraje de consumo voluntario, y sistemas de autorregulación; asimismo, garantiza el acceso a procedimientos administrativos y judiciales ágiles, expeditos y eficaces para la resolución de conflictos y la reparación de daños. Igualmente, facilita el acceso a las acciones por intereses colectivos y difusos. (Código de Protección y Defensa del Consumidor, 2010)

En esta breve descripción de los alcances de la nueva Política de Protección al Consumidor, se observa que, en términos generales, el Estado debe garantizar mecanismos eficaces y expeditivos para la solución de conflictos entre proveedores y consumidores. En atención a ello, se analizará, a continuación, la manera en que el Estado, a través de Indecopi, ha venido promoviendo mecanismos de prevención y 
solución de conflictos, así como su aplicación en los procedimientos de protección al consumidor a su cargo.

\section{FunCión de INDECOPI EN MATERIA DE PROTECCIÓN AL CONSUMIDOR}

Indecopi fue creado en noviembre de 1992 y desarrolla sus funciones de acuerdo con lo dispuesto por el Decreto Legislativo 1033, que aprueba la Ley de Organización y Funciones de Indecopi, así como por el Decreto Legislativo 807, Ley sobre Facultades, Normas y Organización.

Entre las múltiples tareas asignadas a Indecopi en su papel de agencia de competencia, el artículo 2 de la Ley de Organización y Funciones establece expresamente que dicha entidad tiene como función proteger los derechos de los consumidores a través de sus órganos funcionales. Para ello, vigila que la información en los mercados sea la correcta, asegura la idoneidad de los bienes y servicios en función de la información brindada, y evita la discriminación en las relaciones de consumo.

En estas funciones radica la importancia de Indecopi en la defensa del consumidor, lo cual no solo requiere de la capacidad técnica de sus miembros, sino también de un desempeño proactivo, oportuno y eficaz. Por ejemplo, en la literatura jurídica, Kresalja y Ochoa (2012) indican al respecto:

Hay territorios, como el de la protección al consumidor que está a su cuidado, donde no basta ser un árbitro, sino que, en una sociedad con tan poca igualdad como la nuestra, es preciso tener un rol proactivo $y$, en ocasiones, protector para poder edificar un Estado social y democrático de derecho. (p. 271)

Partiendo de dicha premisa, se entienden mejor los motivos por los que Indecopi ha promovido en los últimos meses el diseño de políticas públicas orientadas a brindar una adecuada protección a los derechos de los consumidores, así como la implementación de diversos incentivos que permitan que estos logren una solución más rápida y satisfactoria a sus problemas de consumo.

La nueva visión de Indecopi se relaciona con la promoción de soluciones efectivas y rápidas para los consumidores, lo cual se encuentra en armonía con la dinámica actual del mercado, mediante el diseño de una estrategia en tres niveles: (i) promoción de programas de compliance o cumplimiento normativo en materia de protección al consumidor, 
(ii) promoción de mecanismos de autocomposición y (iii) generación de incentivos para la conclusión anticipada de los procedimientos administrativos de protección al consumidor motivados por denuncia de parte (Gagliuffi, 2017).

De lo anterior, se colige que Indecopi cuenta con una perspectiva de lo que se busca promover en materia de protección al consumidor y a quiénes aspira a beneficiar con dicha actuación. Con este fin, va más allá de la tramitación de procedimientos e imposición de sanciones, y se enfoca en incentivar la existencia de mecanismos que permitan al consumidor obtener una solución efectiva y rápida a sus conflictos de consumo, los cuales se analizarán más adelante. Sin perjuicio de lo anterior, debe considerarse que establecer mecanismos de solución más céleres a favor de los consumidores es una medida que requiere necesariamente la participación de los proveedores, dado que son estos, generalmente, el primer filtro frente a los consumidores, y su actuación a favor de dar una solución hará posible una mayor satisfacción de las necesidades de los destinatarios finales de sus servicios.

Finalmente, es importante que la ejecución de dichas medidas se realice de la mano de una fiscalización continua del mercado en aquellos sectores donde, debido a la baja probabilidad de detección de las conductas infractoras, los proveedores no tengan incentivos suficientes para adecuarse a la normativa, a fin de propiciar mecanismos de solución directa con el consumidor o corregir inmediatamente sus conductas. En esos casos, será vital que Indecopi continúe con la ejecución de un plan de supervisión que sirva como mecanismo disuasivo de la comisión de infracciones a las normas de protección al consumidor por parte de los proveedores; claro, sin perder de vista el objetivo general de la Política Pública que busca incidir en la protección de los consumidores (en especial, del segmento más vulnerable del país).

\section{MeCANismos De SIMPLIFICACIÓN DE LOS PROCEDIMIENTOS ADMinistrativos de PROTECCIÓN AL CONSUMIDOR}

Desde hace muchos años, Indecopi enfrenta una tendencia creciente del número de procedimientos en materia de protección al consumidor, motivados por denuncias de parte. Esto genera una disconformidad en los consumidores respecto a la calidad del servicio, pues perciben una demora excesiva en la obtención de una solución por parte del 
proveedor o un pronunciamiento final sobre su problema de consumo. A fin de revertir esta situación, con la promulgación y entrada en vigencia del Código se instauró el mecanismo denominado procedimiento sumarísimo de protección al consumidor, cuyo objetivo es ser mucho más expeditivo en atención a la "menor cuantía" de los bienes o servicios en controversia, pues resolvería las denuncias de los consumidores en un plazo máximo de treinta (30) días hábiles.

Sin embargo, posteriormente, a través de la Directiva 007-2013/ DIR-COD-INDECOPI, se introdujo una modificación normativa que distorsionó de manera estructural el objeto del referido procedimiento al establecer que la presentación de un desistimiento o acuerdo conciliatorio no surtía los efectos de conclusión del procedimiento una vez iniciado este - lo cual significó una acentuación de su carácter sancionador- y únicamente podría considerarse como una circunstancia atenuante de la sanción por imponer, de ser el caso.

Aunque no se tienen estadísticas al respecto, un análisis directo de este cambio muestra fácilmente que implicó una suerte de retroceso en la política pública de promoción de mecanismos alternativos de solución de conflictos. Esto se debía a que el inicio del procedimiento conllevaba necesariamente el pronunciamiento de fondo de la autoridad, lo que restaba incentivos a los proveedores para que lleguen a acuerdos conciliatorios, en la medida en que estos aún se encontraban expuestos al riesgo de multas, pese a haber tomado medidas inmediatas para satisfacer las expectativas de los consumidores.

En ese sentido, si bien con la promulgación y entrada en vigencia del Código se instituyó el procedimiento sumarísimo a fin de resolver rápidamente gran parte de los conflictos entre consumidores y proveedores, la directiva en mención distorsionó tal objetivo y generó un efecto contraproducente. Así, Indecopi terminó destinando sus recursos a mantener la tramitación de casos individuales que no tenían un impacto trascendente en el bienestar de los consumidores, en general, dado que el consumidor afectado en el caso concreto ya había visto satisfechos sus intereses, según la evidencia de un acta de conciliación o un desistimiento presentado por el propio consumidor.

No obstante, con la publicación del Decreto Legislativo 1308 en el mes de diciembre del 2016, se introdujeron diversas modificaciones al Código orientadas a simplificar los procedimientos de protección al consumidor con el fin de brindar una solución más rápida y eficaz a los 
consumidores. A la vez, esto evitaría mantener a la institución continuando casos en que las afectaciones particulares ya habían sido corregidas o reparadas de algún modo por el proveedor y aceptadas como tal por el consumidor denunciante. Entre los cambios efectuados destacan aquellos que incorporan nuevas formas de conclusión del procedimiento motivado por denuncia de parte, así como una causal de improcedencia de la denuncia en aquellos supuestos donde el proveedor haya subsanado o corregido la conducta con anterioridad a la notificación de cargos.

Para el primer caso, ahora el artículo 107-A del Código establece que, en cualquier estado o instancia del procedimiento de oficio promovido por denuncia de parte, el órgano resolutivo puede declarar su conclusión anticipada en los siguientes supuestos:

a) Cuando el denunciante formule desistimiento del procedimiento o de la pretensión antes de la notificación de la resolución que agota su vía administrativa.

b) Cuando las partes lleguen a un acuerdo mediante conciliación, mediación, transacción o cualquier otro acuerdo que, de forma indubitable, deje constancia de que se ha solucionado la controversia materia de denuncia antes de la notificación de la resolución que pone fin a la misma. (Código de Protección y Defensa del Consumidor, 2010)

Dicho esto, actualmente sí es posible que la autoridad administrativa concluya los procedimientos administrativos motivados por denuncia de parte cuando exista un desistimiento o un acuerdo entre el consumidor y el proveedor, lo que permite que Indecopi pueda destinar sus recursos a la ejecución de fiscalizaciones, acciones de difusión para consumidores y proveedores, así como a otras actuaciones que generen un impacto positivo en el bienestar de los consumidores, en general.

De igual modo, la inclusión de una nueva causal de improcedencia de la denuncia que presente un consumidor cuando el proveedor haya subsanado o corregido su conducta antes de la notificación de cargos - claro está, cuando existen hechos pasibles de subsanación- es un incentivo para que los proveedores corrijan su conducta rápidamente y sin esperar el inicio de un procedimiento por parte de Indecopi. Esto causa que los consumidores se vean satisfechos en sus expectativas de consumo, y son ellos los beneficiarios de este tipo de incentivos. 
Evidentemente, este tipo de medidas constituye un primer paso para la generación de mayores mecanismos que permitan garantizar el respeto de los derechos de los consumidores, sin afectar las acciones de fiscalización y monitoreo continuo de Indecopi mediante sus órganos funcionales. En consecuencia, constituye una mejora sustancial en beneficio de los agentes involucrados en la relación de consumo, al generar incentivos en el proveedor para una corrección de su conducta a fin de evitar los costos de tiempo y recursos inherentes a la continuación del procedimiento, además de ofrecer una alternativa de solución más rápida al consumidor.

\section{Mecanismos JuRídicos Alternativos de SOlución DE CONFLICTOS}

La existencia de mecanismos alternativos de solución de conflictos resulta de suma importancia en el entorno social actual porque promueve el consenso entre las partes de la relación de consumo, de acuerdo con su libre elección, lo que propicia un empleo adecuado de recursos y tiempo tanto para el Estado como para las propias partes involucradas en el conflicto. Por tal motivo, ello no podía quedar ajeno al ámbito de la protección al consumidor y fue introducido a partir del Código a título de política pública, posteriormente reforzada dentro de uno de los ejes de la Política Nacional de Protección al Consumidor, desarrollada previamente.

\section{Conciliación}

En términos generales, la conciliación es un mecanismo de solución de conflictos a través del cual dos o más personas se encargan por sí mismas de la solución de sus diferencias, generalmente, con la ayuda de un tercero neutral, denominado conciliador. Su trascendencia como mecanismo alternativo de solución de conflictos, en todos sus niveles, ha llegado a tal punto que el artículo 1 de la Ley 26872, que regula la conciliación (1997), ha declarado de interés nacional su institucionalización y desarrollo: "Declárese de interés nacional la institucionalización y desarrollo de la Conciliación como mecanismo alternativo de solución de conflictos". De este modo, con la vigencia del Decreto Legislativo 1308, que modifica el Código, Indecopi emitió nuevas directivas - la 006-2017/DIR-COD-INDECOPI y la 005-2017/DIR-COD-INDECOPI-, correspondientes a los procedimientos de protección al consumidor y al 
procedimiento sumarísimo sobre la misma materia, respectivamente, y otorgó nuevamente la importancia que le corresponde a la conciliación como un mecanismo útil para brindar una solución a un conflicto. Ahora esta es aceptada en cualquier instancia del procedimiento, además de que, al igual que en la anterior directiva, se contempla como agravante la inasistencia del proveedor a las audiencias convocadas por el Servicio de Atención al Ciudadano o los órganos resolutivos, lo que es un mayor incentivo para que el proveedor tome medidas prontas para dar solución a un problema de consumo.

Con esta modificación, se espera que Indecopi tome un nuevo impulso a fin de reducir la estadística de denuncias y conduzca sus esfuerzos a aquellos casos que revistan mayor gravedad y, especialmente, generen un mayor impacto en beneficio de los consumidores en general, dado que estos resultan ser la piedra angular de una economía social de mercado.

\section{Libro de Reclamaciones}

Algunos meses antes de la entrada en vigencia del Código, a través del Decreto Supremo 077-2010-PCM, se implementó un mecanismo por entonces novedoso ${ }^{3}$ para la efectiva solución de los conflictos en materia de protección al consumidor: el Libro de Reclamaciones. La premisa, bastante clara, partía del hecho de que proveedores y consumidores se enfrascaban en largos procedimientos para arreglar sus diferencias, debido a la ausencia de una vía formal dinámica para canalizar tanto el descontento del consumidor como la acción de respuesta del proveedor. Para estos efectos, el Libro de Reclamaciones se presenta como el primer intento de solución de la controversia, ya que propicia el contacto directo entre consumidor y proveedor en los establecimientos abiertos al público.

A partir de la aprobación del Código, y con el pasar de los años luego de su reglamentación, fueron introduciéndose modificaciones y mejoras

3 El Libro de Reclamaciones constituyó una importación de los avances realizados en Latinoamérica (por ejemplo, Argentina, Brasil, Guatemala) y Europa (España y Portugal). Para mayor detalle sobre estas experiencias, véase la ponencia "Experiencias comparadas en materia de Libro de Reclamaciones" de Daniel Navarro Reto y Milagros Pozo Ascuña en el XX Congreso Internacional del CLAD sobre la Reforma del Estado y de la Administración Pública, realizado en Lima entre el 10 y el 15 de noviembre del 2015. 
al funcionamiento de este medio, a fin de darle mucha mayor eficacia para prevenir la formulación de controversias ante Indecopi en forma de procedimientos sancionadores y realizar un sondeo del mercado para orientar las intervenciones de la entidad. Con este enfoque, a partir de junio del 2017, entró en vigencia una mejora sustancial a esta herramienta de solución directa de conflictos. Mediante el Decreto Supremo 058-2017-PCM, se modificó el Reglamento del Libro de Reclamaciones para favorecer los acuerdos conciliatorios entre las partes, en los que el Libro actúa como principal evidencia (aunque también se admiten correos electrónicos u otros medios alternativos, dependiendo de cada caso); de igual manera, se establecieron efectos perjudiciales para el proveedor, si este incumpliera.

Estos cambios, por ejemplo, admiten que ahora exista la posibilidad de que el proveedor llegue a un acuerdo directo con el consumidor cuando este consigne un reclamo en el Libro de Reclamaciones; en dicho supuesto, el proveedor deberá dejar constancia expresa de lo ofrecido y el consumidor podrá prestar su conformidad de forma indubitable. De este modo, el referido acto tendrá la naturaleza de un acuerdo conciliatorio entre las partes, y si el proveedor no cumple con lo ofrecido, podrá ser sancionado por incumplimiento de acuerdo conciliatorio y recibir una penalización económica que va desde 1 hasta 200 UIT.

Tales modificaciones obedecen a la necesidad de que el Libro de Reclamaciones amplíe sus ventajas y no se reduzca solamente a un medio para dejar constancia de la disconformidad en el servicio (como hasta ahora venía funcionando), sino que también sea expresión de la coordinación de esfuerzos de las partes para lograr consensos en supuestos de controversia, aunque el impacto del cumplimiento de este objetivo se verá en los siguientes meses y años. Lo que sí resulta claro es que este mecanismo permitirá de manera transversal que los proveedores (incluso los micro y pequeños negocios) tengan la opción de llegar a una solución más rápida al conflicto con el consumidor, sin incurrir en los costos adicionales de tiempo y dinero que podría involucrar su participación en un procedimiento administrativo. Además, para ellos será un medio para identificar directamente cuáles son los aspectos de su negocio que deben corregir a fin de mejorar sus servicios o los productos que ofrecen al público, por lo que resulta una herramienta que contribuye a generar la confianza de los consumidores. 


\section{Arbitraje de consumo}

En el arbitraje, a diferencia de los medios antes mencionados, el conflicto no es solucionado por las partes, sino por un órgano colegiado (el tribunal arbitral), cuyos miembros (árbitros) pueden ser designados directamente por las partes o, en su defecto, por una institución arbitral o la Cámara de Comercio, conforme con lo dispuesto en los artículos 22 y 23 del Decreto Legislativo 1071, que norma el arbitraje en el Perú.

Esta vía de resolución de conflictos fue introducida en el sistema de protección al consumidor por el artículo 137 del Código, en el cual se dispone la creación del Sistema de Arbitraje de Consumo (SISAC). En este punto surge el primer reto, ya que debía llevarse un mecanismo que implicaba altos costos para las partes a un ámbito en el que el Estado impulsa el desarrollo del procedimiento. En esa línea, cabe resaltar que la gratuidad de este tipo de mecanismos es un elemento clave para el funcionamiento óptimo de esta medida.

En efecto, si bien este mecanismo, por su flexibilidad y simplicidad, ha sido diseñado para resolver controversias relacionadas con bienes o servicios de alto valor económico, por lo que ha sido utilizado con mayor intensidad en conflictos entre empresas y Estados tanto en el ámbito nacional como internacional, su naturaleza gratuita en materia de protección al consumidor es un atractivo para las partes involucradas. Por ejemplo, desde la implementación de la Junta de Arbitraje de Consumo, se han recibido 43 solicitudes de arbitraje provenientes de los siguientes sectores: financiero y seguros $(25 \%)$, comercio retail y venta al por menor ( $23 \%)$, equipos de telecomunicaciones y software (12\%), construcción e inmobiliaria $(9 \%)$, servicio de transporte $(7 \%)$, servicios profesionales $(7 \%)$, prestación de servicios personales de peluquería y funerarias (5\%) (Indecopi, 11 de agosto del 2017).

Finalmente, si bien el arbitraje de consumo (dado su carácter sencillo, gratuito, rápido y de cosa juzgada) se está convirtiendo poco a poco en un mecanismo válido y útil para que los proveedores y consumidores obtengan una solución rápida a sus conflictos, es necesario generar mayores canales de difusión sobre las características y beneficios de este modelo para incrementar su demanda actual. 


\section{Programas de cumplimiento normativo en el Ámbito DE LAS RELACIONES DE CONSUMO}

En los últimos años, se ha dado relevancia a los programas de compliance como un mecanismo eficaz para asegurar el adecuado funcionamiento de una empresa en el mercado. En particular, el término compliance o cumplimiento significa, en líneas generales, actuar conforme a la ley, las normas y prácticas buenas. En ese sentido, se ha señalado que "compliance can be defined as the duty to comply, to be in accordance and enforce the laws, guidelines, internal and external rules, aiming to mitigate the risks associated with the reputation and the legal and regulatory risk" (Athay, 2016, pp. 17-18). En atención a lo anterior, puede decirse:

[Por] regulación de compliance se entiende aquella que exige a ciertas personas físicas o jurídicas la creación de un programa que incluya normas y procedimientos internos destinados a prevenir, identificar y corregir actos que violen ciertas regulaciones o ciertos principios voluntariamente adoptados ("programa de compliance"). (Serrano et al., 2015, p. 17)

En virtud de ello, los programas de compliance deberían estar diseñados sobre la base de siete (7) pilares: (i) una cultura del cumplimiento, (ii) el preestablecimiento de objetivos empresariales, (iii) evaluación de riesgos, (iv) adopción de medidas necesarias para la contención de riesgos, (v) delimitación de los ámbitos de competencia, (vi) sistemas internos de comunicación y (vii) sistemas de supervisión y sanción (Coca, 2013, pp. 47-72).

En este punto, cabe destacar que este tipo de programas adquieren, en el ámbito del derecho administrativo, gran relevancia si se tiene en cuenta que la vulneración de la normativa correspondiente puede traer importantes consecuencias jurídicas y económicas para los infractores. Por ejemplo, en el derecho de la competencia, los programas de cumplimiento serios deben ser contemplados como circunstancia atenuante con relación al importe de la multa, dado que de esta forma se crean incentivos para la inversión de medios y dinero en la implementación de programas en el seno de las empresas, de manera que todos los miembros de la organización conozcan los riesgos corporativos y personales de vulnerar esta normativa (Pérez, 2014).

En esa línea, la legislación peruana actual considera fundamental promover programas de cumplimiento normativo en materia de 
protección al consumidor por parte de los proveedores. Tanto es así que el artículo 112 del Código reconoce dicha iniciativa del proveedor y la establece como una circunstancia atenuante en la graduación de la sanción. No obstante, se debe tener en cuenta que este mecanismo no es visto como un incentivo real para todo tipo de proveedores, pues, por las condiciones que requiere su implementación efectiva, parece claro que solo algunas empresas valorarán los beneficios de su ejecución frente al riesgo que genere su incumplimiento.

De este modo, la promoción de una política de cumplimiento normativo constituye una herramienta eficaz para reducir el riesgo de infracciones y el número de conflictos de consumo, pero solo respecto de un sector del mercado que cuenta con una estructura formal y con procesos internos que permitan prevenir, identificar y corregir cualquier incumplimiento de las normas o de sus políticas internas de gestión. Por tal motivo, el fomento de estos mecanismos debe estar acompañado de una labor de monitoreo continuo del mercado que incentive a los agentes económicos a implementar este tipo de programas.

\section{COMENTARIOS FinALES}

La Política Nacional de Protección al Consumidor es un instrumento importante que permite la articulación de los objetivos en materia de protección al consumidor en el ámbito multisectorial. Además, implementa una política pública nacional para la promoción de mecanismos de prevención y solución de conflictos entre consumidores y proveedores, lo cual contribuye a lograr una solución más eficaz a los conflictos de consumo.

Evidentemente, el diseño de esta política pública parte de una adecuada formulación del problema público en materia de protección al consumidor, al identificar la necesidad del consumidor que requiere una solución rápida y eficaz a su conflicto de consumo. Por tanto, se espera que su implementación tenga un impacto positivo en la sociedad.

Respecto al papel de Indecopi, es claro que la simplificación de las normas aplicables a sus procedimientos administrativos de protección al consumidor permitirá viabilizar el cumplimiento de la política pública en cuestión, pues considera a todos los actores involucrados en una relación de consumo y, por tanto, implica un beneficio directo e indirecto tanto a los consumidores como a los proveedores. 
Sin perjuicio de lo anterior, es fundamental que Indecopi mantenga una gestión continua, proactiva y oportuna en sus acciones de fiscalización en los diversos sectores, y que se oriente a prevenir, identificar y corregir las afectaciones que se presenten en diversos segmentos, en especial, entre los consumidores más vulnerables, de acuerdo con el objetivo instaurado en la actual Política Nacional de Protección al Consumidor.

\section{REFERENCIAS}

Athay, A. (2016). Perspectives for the promotion of a competition environment in Brazil. Contribución presentada en Intergovernmental Group of Experts on Consumer Protection Law and Policy, First Session, 17 al 18 de octubre. Ginebra. Recuperado de http://unctad.org/meetings/en/Contribution/ciclp2016c13_Brazil_ CADE_en.pdf

Coca, I. (2013). ¿Programas de cumplimiento como forma de autorregulación regulada? En J.-M. Silva Sánchez (Dir.) y R. Montaner Fernández (Coord.), Criminalidad de empresa y compliance. Prevención y reacciones corporativas (pp. 43-72). Barcelona: Atelier Libros Jurídicos.

Decreto Legislativo 1033, Ley de Organización y Funciones de Indecopi. (25 de junio del 2008). Recuperado del sitio de internet del Sistema Peruano de Información Jurídica: http://spij.minjus.gob.pe/libre/ main.asp

Decreto Legislativo 1071, Ley de Arbitraje. (28 de junio del 2008). Recuperado del sitio de internet del Sistema Peruano de Información Jurídica: http://spij.minjus.gob.pe/libre/main.asp

Decreto Legislativo 807, Ley sobre Facultades, Normas y Organización de Indecopi. (18 de abril de 1996). Recuperado del sitio de internet del Sistema Peruano de Información Jurídica: http://spij.minjus. gob.pe/libre/main.asp

Decreto Supremo 006-2017-PCM, Decreto Supremo que aprueba la Política Nacional de Protección y Defensa del Consumidor. (27 de enero del 2017). Recuperado del sitio de internet del Sistema Peruano de Información Jurídica: http://spij.minjus.gob.pe/libre/ main.asp

Decreto Supremo 077-2010-PCM, Reglamento del Libro de Reclamaciones. (19 de febrero del 2011). Recuperado del sitio de internet del Sis- 
tema Peruano de Información Jurídica: http://spij.minjus.gob.pe/ libre/main.asp.

Donayre Montesinos, C. (2015). La protección del consumidor desde una perspectiva constitucional. Fundamentos, garantías y mecanismos de protección. Praeceptum, 3, 54-55.

Gagliuffi, I. (2 de febrero del 2017). Más soluciones, menos litigios. El Comercio. Recuperado de https://elcomercio.pe/opinion/colabora dores/soluciones-litigios-ivo-gagliuffi-162432

Indecopi. (11 de agosto del 2017). Sistema de Arbitraje de Consumo. A un año de su puesta en servicio, nuevo sistema de solución de conflictos recibió 43 solicitudes de arbitraje. Recuperado de https:// www.indecopi.gob.pe/noticias/-/asset_publisher/E4hIS8IHZWs9/ content/sistema-de-arbitraje-de-consumo-a-un-ano-de-su-puestaen-servicio-nuevo-sistema-de-solucion-de-conflictos-recibio-43solicitudes-de-arbitraje?inheritRedirect=false

Indecopi. (2017). Política Nacional de Protección y Defensa del Consumidor. Plan nacional de protección de los consumidores 2017-2020. Lima: Autor.

Kresalja, B., y Ochoa, C. (2012). El régimen económico de la Constitución de 1993. Lima: Pontificia Universidad Católica del Perú, Fondo Editorial.

Ley 29571, Código de Protección y Defensa del Consumidor. (2 de septiembre del 2010). Recuperado del sitio de internet del Sistema Peruano de Información Jurídica: http://spij.minjus.gob.pe/libre/ main.asp

Ley 26872, Ley de Conciliación. (13 de noviembre de 1997). Recuperado del sitio de internet del Sistema Peruano de Información Jurídica: http://spij.minjus.gob.pe/libre/main.asp

Pérez, P. (2014). La importancia de los programas de cumplimiento -compliance programmes - en las políticas sancionadoras de las autoridades de competencia. ICE: Revista de Economía, 876, 75-90.

Sentencia 01865-2010 (Lima). (20 de julio del 2011). Tribunal Constitucional del Perú. Recuperado de http://tc.gob.pe

Serrano, P., et al. (2015). Práctica de compliance en Latinoamérica. Estado actual de la legislación anticorrupción y otras. Bogotá: Autores Editores. 
